\title{
Design and evaluation of a miniature laser speckle imaging device to assess gingival health
}

Caitlin Regan

Sean M. White

Bruce Y. Yang

Thair Takesh

Jessica Ho

Cherie Wink

Petra Wilder-Smith

Bernard Choi 


\title{
Design and evaluation of a miniature laser speckle imaging device to assess gingival health
}

\author{
Caitlin Regan,, ${ }^{a, b}$ Sean M. White, ${ }^{b}$ Bruce Y. Yang, ${ }^{b}$ Thair Takesh, ${ }^{b}$ Jessica Ho,, ${ }^{b}$ Cherie Wink, \\ Petra Wilder-Smith, ${ }^{b, d}$ and Bernard Choi ${ }^{a, b, d, e, f, *}$ \\ aUniversity of California, Irvine, Department of Biomedical Engineering, 3120 Natural Sciences II, Irvine, California 92697, United States \\ bUniversity of California, Irvine, Beckman Laser Institute, 1002 Health Sciences Road East, Irvine, California 92612, United States \\ 'Concorde Career College, 12951 Euclid Street, Garden Grove, California 92840, United States \\ ¿University of California, Irvine Medical Center, Department of Surgery, 333 City Boulevard West, Suite 700, Orange, California 92868, \\ United States \\ eUniversity of California, Irvine, Edwards Lifesciences Center for Advanced Cardiovascular Technology, 2400 Engineering Hall, Irvine, \\ California 92697, United States \\ 'CHOC Children's Hospital, 1201 West La Veta Avenue, Orange, California 92868, United States
}

\begin{abstract}
Current methods used to assess gingivitis are qualitative and subjective. We hypothesized that gingival perfusion measurements could provide a quantitative metric of disease severity. We constructed a compact laser speckle imaging (LSI) system that could be mounted in custom-made oral molds. Rigid fixation of the LSI system in the oral cavity enabled measurement of blood flow in the gingiva. In vitro validation performed in controlled flow phantoms demonstrated that the compact LSI system had comparable accuracy and linearity compared to a conventional bench-top LSI setup. In vivo validation demonstrated that the compact LSI system was capable of measuring expected blood flow dynamics during a standard postocclusive reactive hyperemia and that the compact LSI system could be used to measure gingival blood flow repeatedly without significant variation in measured blood flow values $(p<0.05)$. Finally, compact LSI system measurements were collected from the interdental papilla of nine subjects and compared to a clinical assessment of gingival bleeding on probing. A statistically significant correlation $(\rho=0.53 ; p<0.005)$ was found between these variables, indicating that quantitative gingival perfusion measurements performed using our system may aid in the diagnosis and prognosis of periodontal disease. ๑ 2016 Society of Photo-Optical Instrumentation Engineers (SPIE) [DOI: 10.1117/1.JBO.21.10.104002]
\end{abstract}

Keywords: laser speckle contrast imaging; laser Doppler flowmetry; temporal speckle contrast; transillumination; intraoral; gingivitis. Paper 160394SSR received Jun. 11, 2016; accepted for publication Oct. 3, 2016; published online Oct. 27, 2016.

\section{Introduction}

Gingivitis is a disease caused by a buildup of dental plaque, a bacterial biofilm, on the teeth and in the gingival sulcus. Symptoms of gingivitis include inflammation of the gingiva, which can result in redness, tenderness, and swelling of the gums, as well as bleeding during brushing or flossing. ${ }^{1-3}$ Current methods to diagnose gingivitis include visual inspection and mechanical probing of the gingiva by a dentist who scores gingival health on a 0 to 3 scale, where 0 corresponds to healthy gingiva and a 3 indicates severe inflammation and bleeding. ${ }^{4,5}$ However, the results of this assessment technique are qualitative and subjective. ${ }^{5}$ Alternatively, enzymatic biomarkers in the saliva and crevicular fluid can be used for diagnosis, but such tests are time-consuming and expensive. ${ }^{6,7}$ We hypothesize that there are associated changes in gingival perfusion that accompany gingivitis, ${ }^{3}$ and that laser speckle imaging (LSI) can be used to quantitatively measure those changes, potentially providing a simple method for objective diagnosis of periodontal disease and evaluation of treatment approaches.

LSI is a widefield technique used to visualize and quantify blood flow. It was developed in 1981 by Fercher and Briers to image vasculature in the retina, ${ }^{8}$ and has since been employed to monitor flow in the brain, skin, and other organs. ${ }^{9-11}$ LSI utilizes image processing techniques to quantify flow with a value known as speckle contrast, which is inversely proportional to the speed and quantity of red blood cells moving through the imaging volume. ${ }^{12}$

A wide variety of LSI system embodiments exist, including clinical systems, benchtop laboratory systems, and microscopybased systems. ${ }^{8-15}$ Such embodiments can range in size from handheld to larger cart-based or tabletop instruments. To enable LSI measurements of the gingiva, the imaging system used must be miniaturized such that it can be easily positioned and stabilized in the oral cavity. Further, to facilitate clinical integration, the system must also be simple to use and perform measurements relatively quickly. Our goal was, therefore, to design a miniature dental LSI probe with the following features: (1) small enough to be securely positioned in the mouth, (2) simple to use, (3) capable of producing accurate blood flow measurements, (4) sufficiently rapid to collect data within $5 \mathrm{~min} /$ measurement, and (5) able to provide a quantitative metric correlated to disease.

A number of challenges exist that are associated with creating a compact LSI system for use in the oral cavity. First, each component must be small enough to fit relatively comfortably in the mouth. Furthermore, all electrical components must be housed to keep them dry and free of saliva. Additionally, they must be able to withstand sterilization between subject measurements. Finally, without affixing the imaging components to the teeth or gingiva, 
significant motion artifact may be introduced, making handheld systems or external imaging systems potentially untenable. We previously published on the ability to measure pulsatile blood flow in the teeth using a fiber bundle-based LSI device that required each subject to hold their head steady while biting down on a laser fiber. ${ }^{14}$ However, this system was difficult or uncomfortable for some subjects and hence may not be suitable for widespread clinical use.

Here, we describe an LSI system that uses components small enough to be mounted completely within the oral cavity. This was accomplished by using a miniature CMOS camera (volume of $\sim 2 \mathrm{~mm}^{3}$ ) and a compact laser diode package that could be fixed in place within the mouth using custom molds fabricated from dental impression putty. Using these molds, the laser diode and camera could be mounted on the buccal and lingual side of the gingiva and be used to perform LSI in a transillumination geometry. ${ }^{14,16}$

Using this system, we performed in vitro experiments to assess the stability, precision, and repeatability of our device to collect speckle contrast data. We additionally performed an in vivo pilot study using our system to measure perfusion in the gingiva above the upper incisors in subjects diagnosed with mild to severe gingivitis, and correlated the measured speckle contrast values with clinical assessments.

\section{Materials and Methods}

\subsection{Use of Human Subjects}

All subject measurements were approved by the Institutional Review Board of the University of California, Irvine.

\subsection{Device Design}

The compact camera used in our LSI system is an Awaiba NanEye CMOS camera (CMOSIS, Raleigh, North Carolina). It is equipped with a f/\# 2.7 lens, which has an 8- to $75-\mathrm{mm}$ depth of focus and utilizes a rolling shutter during image acquisition. The entire package (camera and lens) measures $1.0 \times 1.0 \times 1.7 \mathrm{~mm}^{3}$, which allows for comfortable intraoral use. The NanEye has a 4-pin connection to a USB2 evaluation board that connects to a PC running provided software (Awaiba Viewer v2.15.2.1). To protect the camera and camera wiring, it was secured in a three-dimensional (3-D)-printed casing using hot melt adhesive. The case also provided additional material with which to stabilize the camera during use. We calculated a ratio of $\sim 1.93$ speckles/pixel with this current configuration, which is very close to the Nyquist criteria for speckle sampling. ${ }^{17,18}$ A 90-mW, 785-nm, single-longitudinal mode, 5.6-mm diameter TO can package laser diode (Roithner LaserTechnik GmbH, Vienna, Austria) was used as our coherent light source. The laser diode was powered by the LDC500 laser diode controller (ThorLabs Inc, Newton, New Jersey). The diode was housed within a custom machined cylindrical casing fabricated from delrin. The casing also contained an aluminum heat sink to aid in diode stabilization.

\subsection{Image Processing}

During data collection, "color reconstruction" and "reduce processing" options in the Awaiba Viewer software were turned off and on, respectively, to ensure raw data were collected. The proprietary Awaiba Viewer software saved data as audio video interleave files, which were converted to a tagged image file format (TIFF) sequence using ImageJ software (NIH), followed by computing speckle contrast maps from each image using MATLAB (MathWorks, Natick, Massachusetts). Temporal speckle contrast was calculated as the standard deviation over the mean intensity in each pixel across a series of images. ${ }^{19} \mathrm{We}$ used a rolling temporal contrast value calculated by computing temporal contrast across each pixel of subsequent four-frame sequences ${ }^{14}$ (i.e., frames $1: 4,2: 5$, and $3: 6$ ). We then averaged the contrast within a user-selectable region of interest (ROI) pertaining to the gingival tissue of interest.

\subsection{Static In Vitro Device Validation}

To test the stability of the NanEye camera and the laser diode to be used during in vivo data collection, we measured a $1 \%$ Intralipid solution (Baxter Healthcare, Deerfield, Illinois) over a period of 3 min using the NanEye camera to quantify the changes in speckle contrast not resulting from direct movement or flow. Image sequences were collected at 30 frames per second (fps) with an exposure time of $5 \mathrm{~ms}$. A $70 \times 70$ pixel ROI was chosen to compute the temporal speckle contrast. This measurement was performed at several different intensities ( 9 to 155 counts) resulting from different currents applied to the laser diode, spanning the dynamic range of the NanEye camera to determine the dependence of illumination intensity on measured speckle contrast. We computed the coefficient of variation $(\mathrm{CV}$, equal to standard deviation/mean) across the 3-min data sets to assess the stability of our system.

\subsection{Dynamic In Vitro Device Validation}

We performed in vitro experiments to validate the NanEye camera for use in LSI. A flow phantom was fabricated using polydimethylsiloxane (PDMS) with titanium dioxide added to give a reduced scattering coefficient of $1 \mathrm{~mm}^{-1}$, which is typical of tissue. ${ }^{20}$ A glass microchannel with a diameter of $650 \mu \mathrm{m}$ was embedded in the surface of the PDMS, and $1 \%$ Intralipid was infused at different speeds $(0.25,0.5$, and 1.0 to $5.0 \mathrm{~mm} / \mathrm{s}$ in $1 \mathrm{~mm} / \mathrm{s}$ increments) using a syringe pump (Harvard Apparatus, Holliston, Massachusetts).

We performed the in vitro validation imaging by comparing the NanEye camera and a monochrome Chameleon3 camera (Point Grey, Richmond, BC, Canada), a research grade CCD camera equipped with a 60-mm Nikkor Micro Lens (Nikon, Melville, New York) with f/\# 2.8. We coupled the diode from our device to a beam expander and a ground glass diffuser to diffusely illuminate the flow phantom, and collected sequences of reflectance speckle images with the NanEye and Chameleon3 cameras. Images were collected using an exposure time of $5 \mathrm{~ms}$, and a frame rate of 30 fps. Temporal speckle contrast was computed in a $7 \times 66$ pixel region within the center of the microchannel for $\sim 200$ frames, and then averaged to improve the signal to noise ratio.

\subsection{In Vivo Data Collection Setup}

Prior to the imaging of each subject, we created a custom-fit impression mold of the upper anterior teeth and gingiva. All measurements were taken from the gingiva above the upper incisors. The molds were made with $\sim 50 \mathrm{~g}$ of Zetalabor C-silicone (Hard 85 Shore A) polysiloxane dental impression material (Zhermack Inc., River Edge, New Jersey) combined 
with $\sim 1$ g of Indurent gel catalyst (Zhermack Inc., River Edge, New Jersey). The dental putty and catalyst were mixed by hand for $\sim 60 \mathrm{~s}$ and then formed into a rectangular block of $\sim 2 \times 2 \times 6 \mathrm{~cm}^{3}$. We created an impression of the subject's teeth within a block of dental putty, while pressing firmly to create a close fit around the teeth and gingiva. The putty was held in place until it hardened ( $60 \mathrm{~s})$.

To enable mounting of the encased camera and laser diode, holes were drilled into the buccal and lingual sides of the mold such that a straight light path was created between the light source and the detector for maximum light throughput in a transillumination geometry. The camera and laser diode housings were inserted into the drilled holes and were held rigidly in place via friction. The total cost of each mold was $<2$, and required $\sim 10 \mathrm{~min}$ to complete.

The encased camera and laser diode were wrapped with disposable Cover-All barrier film (Kerr TotalCare, Orange, California) before use on each subject to prevent cross contamination. Additionally, they were sanitized using sterile alcohol prep pads (Dynarex, Orangeburg, New York) between uses.

\subsection{In Vivo Exposure Time Selection}

To aid in the selection of an exposure time to be used during LSI that would maximize sensitivity to blood flow changes during the cardiac cycle, we used the setup described above to measure gingival blood flow while varying the Naneye exposure time from 2 to $23 \mathrm{~ms}$ (corresponding to exposure settings in Awaiba Viewer of 25 to 249 in 24 or 25 unit steps). Thirty seconds of data were collected at each exposure time with minimal perturbation introduced between measurements to minimize vasoconstriction or vasodilation mediated changes in blood flow during the measurement period. Temporal speckle contrast was computed from each data set as described above and the average change in contrast between systole and diastole was computed. This metric was used to quantify the sensitivity of the imaging system to physiologically relevant changes in blood flow at various exposure times. ${ }^{21}$

\subsection{In Vivo Device Validation}

To ensure that we could detect physiological changes in perfusion, we measured the speckle contrast while artificially perturbing blood flow. We used our device to measure flow in the index finger, and performed an arterial occlusion using a pressure cuff. A 90-s arterial occlusion was performed by inflating a pressure cuff to $220 \mathrm{mmHg}$ to stop blood flow to the finger, followed by a 90 -s recovery period.

We also measured baseline perfusion in three regions of the gingiva, moving progressively from the interdental papilla between the upper incisors to the marginal gingiva, to the attached gingiva to demonstrate changes in perfusion due to different gingival physiologies.

\subsection{In Vivo Device Repeatability}

To quantify the repeatability of our device to measure the same region each time the imaging system was inserted into the oral cavity, as well as to quantify the repeatability of our imaging system longitudinally, we acquired three measurements (3-min each) on six subjects every day for five consecutive days. Between measurements, we removed and reinserted the imaging device. Collected image sequences were used to compute temporal contrast within an ROI of $66 \times 66$ pixels corresponding to the interdental papilla above the upper incisors. We computed the average speckle contrast during each 3-min data set, as well as the standard deviation of the contrast across the fifteen measurements collected throughout the week.

\subsection{In Vivo Experimental Measurements}

We recruited 12 subjects at the Concorde Career College Dental Hygiene Clinic, and collected a set of three measurements for 3-min each at $30 \mathrm{fps}$ to ensure stability of the signal. Subject measurements were taken with an exposure time of $5 \mathrm{~ms}$. The average irradiance during patient measurements was $0.095 \mathrm{~W} / \mathrm{cm}^{2}$ with a maximum of $0.24 \mathrm{~W} / \mathrm{cm}^{2}$, which is less than the maximum permissible exposure for tissue of $0.30 \mathrm{~W} / \mathrm{cm}^{2} .^{22}$ Before LSI data were collected, an examination was performed by a dentist to quantify each subject's bleeding on probing index for the marginal gingiva of the upper anterior teeth (6 to 11). We excluded three patients from the final study ( $n=2$ had missing upper anterior teeth, $n=1$ had hardware malfunction during image collection) leaving nine subjects. The average temporal laser speckle contrast was calculated in an ROI corresponding to the interdental papilla for each measurement. We summed the bleeding on probing index for the upper anterior teeth to grade the overall gingival health of each subject. We computed the Spearman's rank correlation coefficient between the average temporal contrast value and the summed bleeding on probing index. We performed a hypothesis test on a linear fit to these data to test the significance of the slope.

\section{Results}

\subsection{Device Design}

We built a device to acquire laser speckle images of the gingiva as shown in Fig. 1. We collected a series of transilluminated images of the gingiva using a miniature camera [Fig. 1(c)] placed on the labial side of a custom-fit dental putty mold [Fig. 1(a)]. We typically collected images of the interdental papilla; however, we also measured perfusion in the attached and marginal gingiva [Fig. 1(b)]. The speckle pattern is generated using a near-infrared laser diode [Fig. 1(c)] on the lingual surface of the mold, which has good depth penetration into perfused tissue. ${ }^{23}$ The mold fits securely around the upper anterior teeth, which holds the camera and laser diode fixed relative to each other and in the same location every time the mold was inserted. We determined that an exposure time of $5 \mathrm{~ms}$ maximized our temporal contrast sensitivity to physiological changes in flow due to the heartbeat; therefore, this exposure time was chosen for all future measurements.

\subsection{Static In Vitro Device Validation}

To monitor the stability of the laser diode intensity, as well as the temporal contrast in images collected using the NanEye camera, we measured a nonflowing liquid phantom over $3 \mathrm{~min}$. The $\mathrm{CV}$ of the intensity remained below $1 \%$ for intensities above $\sim 20$ counts. The $\mathrm{CV}$ of the temporal contrast was $<1 \%$ at intensities between 20 and 140 counts. 


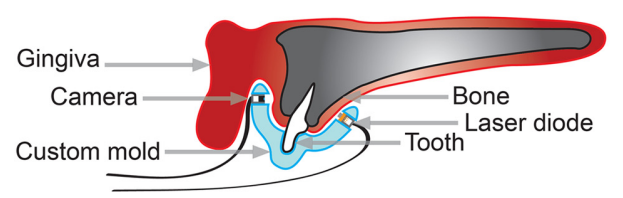

(a)

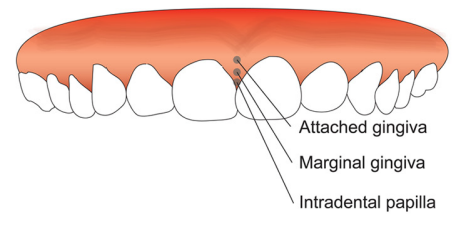

(b)

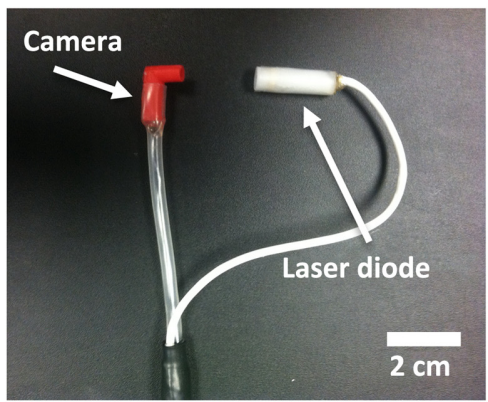

(c)

Fig. 1 Design of miniature intraoral LSI device. (a) The NanEye camera and 785-nm laser diode were placed in a custom-fit dental putty mold, which held them securely in place to acquire transilluminated images of the gingiva. (b) We primarily imaged the interdental papilla between the upper incisors. Images of the marginal and attached gingiva were also acquired to determine if we were sensitive to spatial variations in gingival perfusion. Clinical assessments of bleeding on probing were acquired for the upper anterior teeth (incisors and canines, teeth 6 to 11). (c) The NanEye camera was housed in a 3-D-printed case (red) and the laser diode mounted in a delrin case (white) that contained an aluminum heatsink to ensure intensity stability.

\subsection{Dynamic In Vitro Device Validation}

To ensure the miniature camera was capable of collecting data with sufficient dynamic range and spatial resolution, and with limited artifacts due to the built-in lens system, we compared in vitro flow phantom data collected with a research grade Chameleon3 CCD camera [Fig. 2(a)] to data collected with the NanEye camera [Fig. 2(b)]. We measured the temporal contrast of flow in a glass microcapillary tube at speeds from 0.25 to $5.00 \mathrm{~mm} / \mathrm{s}$ [Fig. 2(c)]. The image from the NanEye camera had lower resolution compared with the Chameleon3 [Fig. 2(a) versus 2(b)], which resulted in lower fidelity object boundaries and an overall grainier image. There was some barrel distortion present near the edges of the images taken with the NanEye camera; however, this did not affect our device due to the fact that the camera is contained within a tube, which inherently reduces the available field of view to the center of the image [Fig. 2(b)].

We demonstrated that contrast decays exponentially with flow speed, as predicted by speckle theory ${ }^{12}$ [Fig. 2(c)]. The dynamic range for temporal contrast using the NanEye was $\sim 0.08$ over the flow speeds we tested and $\sim 0.15$ for the Chameleon3 [Fig. 2(c)]. We observed a linear trend $\left(R^{2}=0.9992\right)$ between contrast values at different flow speeds calculated from images taken with the NanEye versus the Chameleon3 [Fig. 2(d)]. This demonstrated that even though the dynamic range was lower for the NanEye, it still maintained the same contrast response to flow speed as a research grade camera.

To determine the range of intensities where our LSI system remained stable, we measured the temporal contrast as a function of intensity [Fig. 3(a)] in a nonmoving liquid phantom. We observed inflection points in the contrast response at $\sim 20$ and $\sim 140$ counts. Therefore, we maintained the intensity within this range during in vivo clinical data collection.

\subsection{In Vivo Device Validation}

To ensure our device could differentiate between induced changes in in vivo blood flow, we performed an arterial occlusion while collecting measurements in the index finger using our device [Fig. 3(b)]. The occlusion was in place for the first $\sim 20$ s of imaging; during which the average temporal contrast was 0.57. Once the occlusion was released (at $\sim 20 \mathrm{~s}$ ), we observed a reactive hyperemia response for $\sim 5 \mathrm{~s}$ as flow rushed back into the digit causing a marked decrease in contrast. The contrast then settled to a stable baseline of 0.37 for the remainder of the measurement as flow returned to baseline.

To determine the effects of spatial variations in sensor placement, we measured temporal speckle contrast in three locations of the gingiva between the upper incisors of a human subject: the interdental papilla, the marginal gingiva, and the attached gingiva [Fig. 1(b)]. As we moved further away from the interdental papilla, the contrast decreased by 0.013 (Table 1).

\subsection{In Vivo Device Repeatability}

To measure the stability of our system to obtain repeatable contrast values across multiple longitudinal measurements, we measured contrast in the interdental papilla region of the gingiva of six subjects over a period of five consecutive days. Additionally, we were concerned if significant physiological variations in flow occurred from day to day. We collected three data sets from each subject each day and removed the device between each measurement to test the repeatability of our device between uses. We measured an average $\mathrm{CV}$ among the three daily measurements of $6.7 \%$. To determine if daily variations in flow were a significant concern, we computed the standard deviation across the 15 total measurements for each subject (Table 2). One measurement was removed from subjects 1 and 6, and two from subject 5 due to the contrast value being a statistical outlier, which we postulate was due to a problem with the acquisition control software. We assumed that the gingival health was constant in each of the six repeatability subjects over the course of the five daily measurements. We compared the measurements to the standard deviation of the 27 total measurements collected from subjects with varying degrees of gingival health (Concorde Clinic, $\sigma=0.021)$. There was a significant difference in the variance of the repeatability subjects compared to the variance of the group of clinical subjects with different stages of gingivitis ( $p<0.05$, Levene's test for equal variance) (Table 2$)$. This analysis indicates that any variance that occurs from day to day due to the device or to physiological changes in flow is significantly less than the changes we observe due to differences in gingival health and inflammation. 


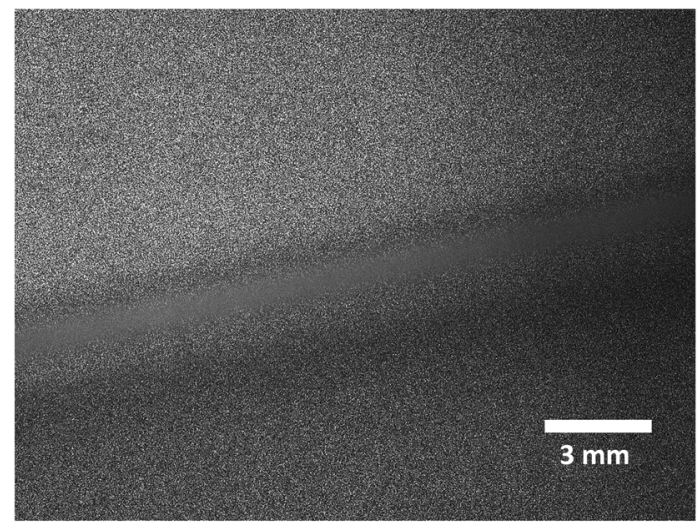

(a)

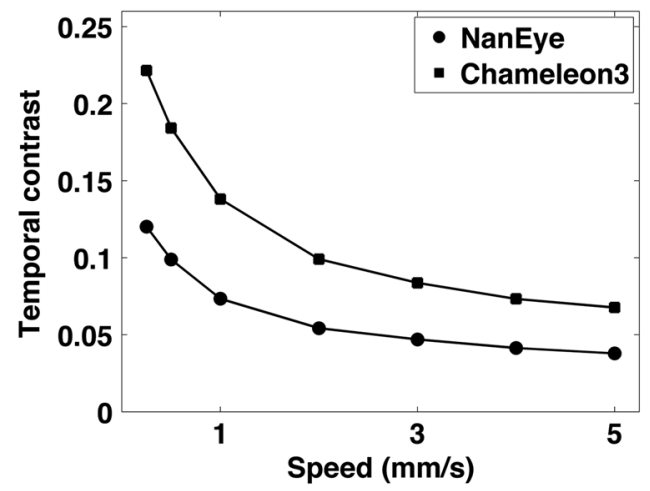

(c)

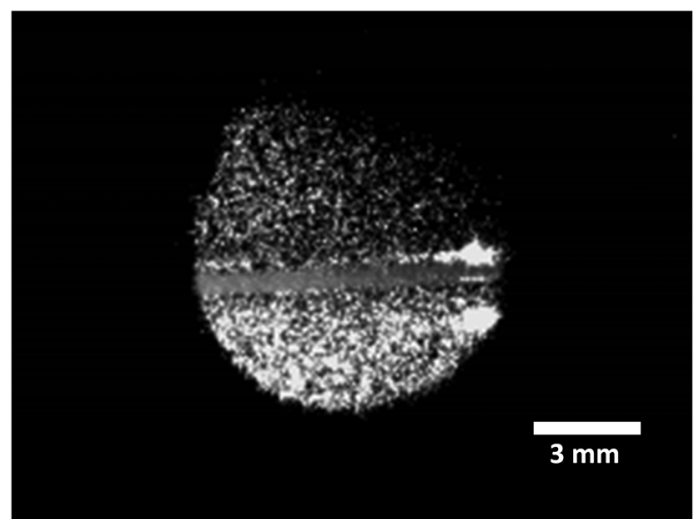

(b)

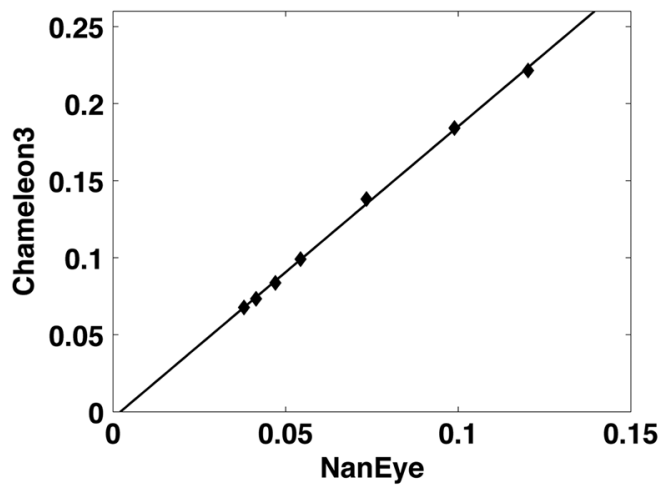

(d)

Fig. 2 Dynamic in vitro device validation. (a) Sample raw speckle image of flow in a glass microchannel (blurred rectangular region) acquired with a research grade CCD camera (Chameleon3, Pt. Grey). (b) Sample raw speckle image of flow in a glass microchannel (blurred rectangular region) acquired with the NanEye camera. The field of view was limited because the camera was encased in a protective housing. (c) Temporal contrast measured simultaneously on flow at different speeds. The dynamic range of the Chameleon3 was greater than that of the NanEye $(0.15$ compared to 0.08$)$. (d). Comparison of temporal contrast at different flow speeds for the Chameleon3 and NanEye cameras resulted in a linear relationship $\left(R^{2}=0.9992\right)$, suggesting the ability of the NanEye to measure blood flow in a comparable manner to the Chameleon3.

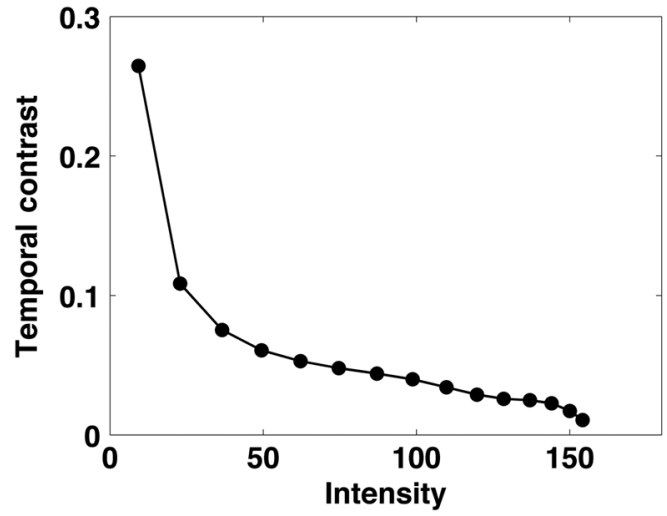

(a)

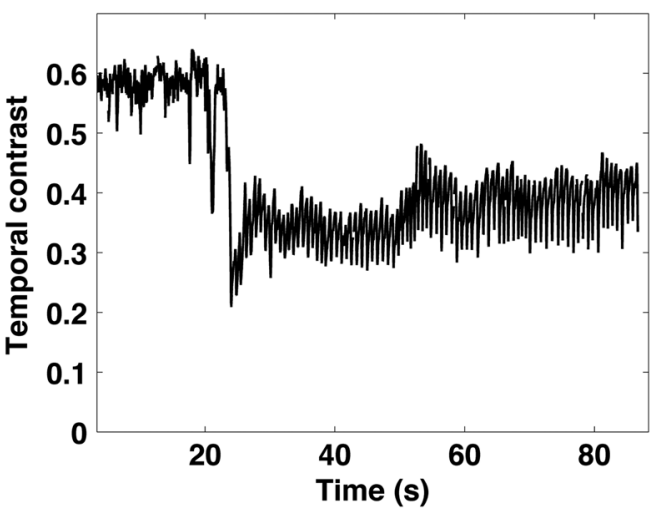

(b)

Fig. 3 (a) We measured temporal contrast as a function of intensity to determine the range of stable contrast measurements. Due to the inflection points at $\sim 20$ and $\sim 140$ counts, we collected all further experimental data with intensities ranging between those values. The CV of the intensity and contrast data were $<1 \%$ in this range. (b) Measurements of physiological changes in blood flow during an arterial occlusion. The flow was low $(K=0.57)$ until the occlusion was released at $\sim 20 \mathrm{~s}$. A reactive hyperemia response occurred for $\sim 5 \mathrm{~s}$ and then flow stabilized to baseline $(K=0.37)$. 
Table 1 Speckle contrast in different spatial locations within the gingiva. Contrast decreased by 0.013 as the probe was moved from the interdental papilla to the attached gingiva.

\begin{tabular}{lc} 
Location & Temporal contrast \\
\hline Interdental papilla & 0.042 \\
Marginal gingiva & 0.031 \\
Attached gingiva & 0.029 \\
\hline
\end{tabular}

Table 2 Three data sets were acquired each day for five consecutive days from six subjects to determine the repeatability of our device; we assume the health of each subject remained constant across the week. The standard deviation of the 15 measurements for each subject is listed, as well as the standard deviation across all measurements taken from subjects with varying degrees of gingival health (Concorde Clinic). Levene's test for equal variance was applied to compare the variance of each subject to the collective data from Concorde Clinic, and the variances were significantly different $(p<0.05)$. This indicates that the variations in contrast due to daily changes in perfusion and instrument noise are significantly less than variations in flow due to different degrees of gingival health.

\begin{tabular}{lcc} 
Subject & Standard deviation $(\sigma)$ & $\begin{array}{c}p \text {-value of } \sigma_{\text {repeatability }} \\
\text { compared to } \sigma_{\text {Concorde }}\end{array}$ \\
\hline Repeatability 1 & 0.008 & 0.008 \\
Repeatability 2 & 0.007 & 0.004 \\
Repeatability 3 & 0.014 & 0.048 \\
Repeatability 4 & 0.011 & 0.015 \\
Repeatability 5 & 0.008 & 0.009 \\
Repeatability 6 & 0.011 & 0.021 \\
Concorde Clinic & 0.021 & - \\
\hline
\end{tabular}

\subsection{In Vivo Experimental Measurements}

Finally, to test our ability to correlate LSI data with gingival health, we took three 3-min measurements in nine subjects with mild to severe gingivitis. We computed a CV of $<14 \%$ between the three measurements for each subject. The CV across all 27 clinical subject measurements was over $34 \%$. We measured the Spearman correlation between the sum of the bleeding on probing index across the frontal and lingual side of the upper anterior teeth and the measured temporal contrast value. We observed a decrease in contrast as the sum of the anterior bleeding on probing score increased (Fig. 4), indicating an increase in flow with decreasing gingival health. The correlation had a Spearman coefficient $(\rho)$ of $-0.53(p<0.005)$, and a linear hypothesis test indicated that the slope was significantly different from zero $(p<0.01)$.

\section{Discussion}

Our experiments collectively demonstrate the feasibility and stability of a miniature LSI device designed for use in the mouth. We built a device that fit securely around the subject's teeth to hold a small camera and compact laser diode in place to

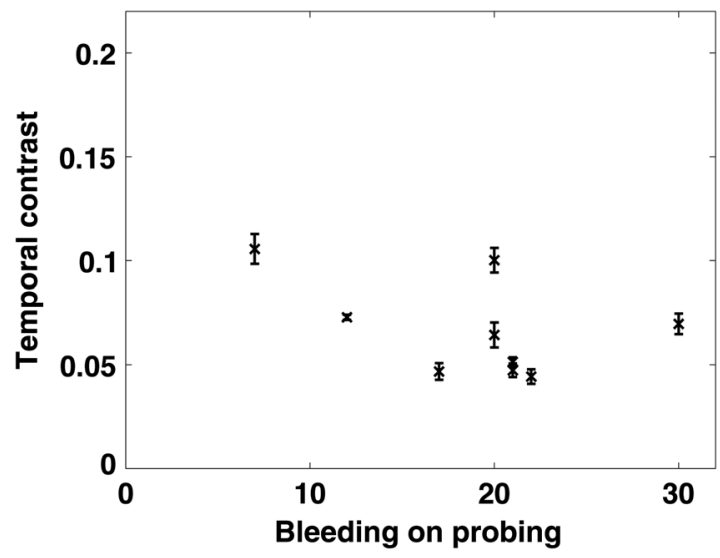

Fig. 4 Three measurements were collected from nine subjects with varying degrees of gingival inflammation. The sum of the bleeding on probing scores ( $x$-axis) for the anterior teeth was correlated to the measurements of temporal contrast ( $y$-axis). We observed a significant increase in perfusion (decrease in contrast) with an increase in bleeding on probing (indicative of worse gingival health) $(\rho=-0.53$, $p<0.005)$. The average temporal contrast with standard deviation is shown for each of the nine subjects.

transilluminate the gingiva (Fig. 1). The impression mold held our imaging system securely in place, and enabled repeated imaging of the same location within the gingiva. This was important to minimize motion artifacts, which hinder accurate clinical data collection in many LSI devices, ${ }^{14}$ as well as to ensure measurements were taken in the same location, which is important because blood flow in the oral cavity may have significant spatial variance due to physiology. ${ }^{24}$

We computed temporal speckle contrast values to obtain an estimate of perfusion in the gingiva. We chose to use the temporal algorithm to compute speckle contrast rather than the spatial algorithm because the former has been shown to be more reliable when quantifying contrast in regions containing static scattering. ${ }^{25}$ Since the detected light likely propagated through static scattering structures such as the roots of the teeth, or even bone, we chose to use the temporal algorithm to maximize our sensitivity to blood flow. This results in relatively low-temporal contrast values $(0.02$ to 0.12$)$ observed in in vivo gingiva (Tables 1 and 2, Fig. 4), which is expected due to the high level of perfusion present in this tissue. We do not observe a significant correlation between speckle contrast computed with the spatial algorithm and bleeding on probing $(\rho=-0.19$, $p>0.35)$, which supports the hypothesis that the temporal algorithm is required to reduce the effect of static scattering due to interpatient physiological variability.

We performed several in vitro and in vivo experiments to test the stability and repeatability of our device. We monitored the stability of the laser diode intensity to ensure that fluctuations in intensity, as well as in temporal contrast, had a CV below $1 \%$. These results indicate that the aluminum heat sink in our device was sufficient for thermal stabilization of the laser diode. We determined that the most stable contrast values were obtained when data were collected with intensity counts between 20 and 140 [Fig. 3(a)]. Additionally, we determined that $5 \mathrm{~ms}$ was the optimal exposure time for our device to be sensitive to physiological changes in flow.

A comparison of our device and a laboratory grade LSI system demonstrated a linear relationship between contrast values from each system as a function of flow speed [Fig. 2(d)]. 
We did observe a lower dynamic range with the miniature oral device [Fig. 2(c)], which is potentially due to the NanEye's comparatively smaller sensor dynamic range ( 72 versus $42 \mathrm{~dB}$ ) resulting from greater noise and reduced full well capacity.

We collected in vivo data that demonstrated the ability of our device to detect changes in perfusion. During an arterial occlusion, we detected a sharp increase in temporal speckle contrast [Fig. 3(b)], consistent with expectations of decreased flow during an occlusion. When the occlusion was released, we observed a characteristic decrease in contrast due to the reactive hyperemia response, before the flow returned to baseline. These data demonstrate that our imaging system was sensitive to induced physiological changes in perfusion.

We also observed a decrease in contrast as we changed the measurement location from the interdental papilla toward the marginal and attached gingiva (Table 1). We postulate that this is due to the corresponding increase in the thickness of the measured region, and hence an increase in the amount of perfusion, causing a decrease in contrast. This highlights the importance of maintaining a repeatable imaging location between measurements, which was facilitated with the mold design used in this study.

We performed in vivo measurements on six subjects to ensure that our device could obtain repeatable contrast values across multiple measurement sessions. The mold is capable of being repeatedly inserted and removed from the subject's mouth while maintaining a similar imaging location for every measurement. Repeatability of imaging locations is critical for future longitudinal studies of gingival blood flow dynamics. Our data demonstrate that the device can be removed and replaced while maintaining measurements of temporal contrast in the gingiva with an average $\mathrm{CV}$ of $6.7 \%$. In comparison, the $\mathrm{CV}$ among measurements in multiple subjects with varying degrees of gingival inflammation is over $34 \%$. Additionally, data collected longitudinally from six repeatability subjects showed significantly less variance $(p<0.05)$ than the measurements collected from subjects in varying stages of gingival health (Table 2). This indicates that not only is our device capable of repeatedly measuring the same location in the gingiva, but that physiological changes in flow from day to day are less than the changes we observe due to variations in gingival health. This result supports our hypothesis that LSI can be used as a diagnostic aid or a tool for the assessment of changes in gingival health over time.

Finally, we performed measurements in subjects that were evaluated for gingivitis symptoms. We acquired similar speckle contrast values in each of the three measurements collected per subject $(\mathrm{CV}<14 \%)$, which is consistent with the previous in vivo validation measurements. Our data show that speckle contrast decreased as the bleeding on probing index for the upper anterior gingiva increased (Fig. 4), which is indicative of more severe gingivitis. We postulate that the decrease in speckle contrast is due to an increase in blood flow, which may be due to increased inflammation in the gingiva, dilation of the microvasculature, or increased vascular density, all of which have been shown to increase in perfusion. ${ }^{3}$

In addition to gingivitis, there are other conditions that affect the oral mucosa. For example, LSI may be used to provide a quantitative assessment of oral mucositis, an inflammatory condition causing painful ulcers in the mouth following chemotherapy and radiation treatments for cancer. ${ }^{26}$ Furthermore, the question of assessing pulpal vitality by detecting blood flow in the pulpal chamber is still unresolved. Our mouth-guard-based device may provide a more stable method of collecting LSI data as compared to previous measurement systems, ${ }^{14}$ and for more specifically targeting locations on the tooth that correspond to the pulpal chamber to reduce contamination of the signal from gingival blood flow.

In conclusion, our in vitro and in vivo data collectively support our hypothesis that LSI can be used to quantitatively measure changes in perfusion associated with gingival health. With continued refinement and evaluation of this platform, we anticipate development of a simple method for objective diagnosis of periodontal disease and evaluation of treatment approaches for gingivitis and other oral microvascular diseases.

\section{Acknowledgments}

The authors would like to acknowledge the support from the Arnold and Mabel Beckman Foundation, the National Institutes of Health (Nos. R01 DE022831 and R01 HD065536), the National Institutes of Health Laser Microbeam and Medical Program (No. P41 EB015890), the National Science Foundation BEST IGERT Program (No. DGE 1144901), and the Roche/ARCS Scholar Award program.

\section{References}

1. M. G. Newman et al., Carranza's Clinical Periodontology, Saunders, St. Louis (2012).

2. G. J. Parfitt, "A five year longitudinal study of the gingival condition of a group of children in England," J. Periodontol. 28(1), 26-32 (1957).

3. H. Zoellner, C. C. Chapple, and N. Hunter, "Microvasculature in gingivitis and chronic periodontitis: disruption of vascular networks with protracted inflammation," Microsc. Res. Tech. 56(1), 15-21 (2002).

4. S. G. Ciancio, "Current status of indices of gingivitis," J. Clin. Periodontol. 13(5), 375-378 (1986).

5. S. H. Wei and K. P. Lang, "Periodontal epidemiological indices for children and adolescents: I. gingival and periodontal health assessments," Pediatr. Dent. 3(4), 353-360 (1981).

6. B. Syndergaard et al., "Salivary biomarkers associated with gingivitis and response to therapy," J. Periodontol. 85(5), 295-303 (2014).

7. S. P. Barros et al., "Gingival crevicular fluid as a source of biomarkers for periodontitis," Periodontol. 2000 70(1), 53-64 (2016).

8. A. F. Fercher and J. D. Briers, "Flow visualization by means of singleexposure speckle photography," Opt. Commun. 37(5), 326-330 (1981).

9. A. K. Dunn et al., "Dynamic imaging of cerebral blood flow using laser speckle," J. Cereb. Blood Flow Metab. 21(3), 195-201 (2001).

10. B. Yang et al., "Intraoperative, real-time monitoring of blood flow dynamics associated with laser surgery of port wine stain birthmarks," Laser. Surg. Med. 47(6), 520-525 (2015).

11. C. Sturesson et al., "Laser speckle contrast imaging for assessment of liver microcirculation," Microvasc. Res. 87, 34-40 (2013).

12. D. A. Boas and A. K. Dunn, "Laser speckle contrast imaging in biomedical optics," J. Biomed. Opt. 15(1), 011109 (2010).

13. S. M. White et al., "Longitudinal in vivo imaging to assess blood flow and oxygenation in implantable engineered tissues," Tissue Eng. Part. C: Methods 18(9), 697-709 (2012).

14. C. Regan et al., "Fiber-based laser speckle imaging for the detection of pulsatile flow," Laser. Surg. Med. 47(6), 520-525 (2015).

15. R. Farraro, O. Fathi, and B. Choi, "Handheld, point-of-care laser speckle imaging," J. Biomed. Opt. 21(9), 094001 (2016).

16. C. Stoianovici, P. Wilder-Smith, and B. Choi, "Assessment of pulpal vitality using laser speckle imaging," Laser. Surg. Med. 43(8), 833837 (2011).

17. S. J. Kirkpatrick, D. D. Duncan, and E. M. Wells-Gray, "Detrimental effects of speckle-pixel size matching in laser speckle contrast imaging," Opt. Lett. 33(24), 2886-2888 (2008).

18. J. C. Ramirez-San-Juan et al., "Effects of speckle/pixel size ratio on temporal and spatial speckle contrast analysis of dynamic scattering 
systems: implications for measurements of blood-flow dynamics," Biomed. Opt. Express. 4(10), 1883-1889 (2013).

19. H. Cheng, Y. Yan, and T. Q. Duong, "Temporal statistical analysis of laser speckle images and its application to retinal blood-flow imaging," Opt. Express. 16(14), 10214 (2008).

20. F. Ayers et al., "Fabrication and characterization of silicone-based tissue phantoms with tunable optical properties in the visible and near infrared domain," Proc. SPIE 6870, 687007 (2008).

21. S. Yuan et al., "Determination of optimal exposure time for imaging of blood flow changes with laser speckle contrast imaging," Appl. Opt. 44(10), 1823-1830 (2005).

22. Office for Research Safety, Northwestern University, "Laser safety handbook," Northwestern University Office for Research Safety, 2011, http://www.research.northwestern.edu/ors/forms/laser-safety-handbook .pdf (January 2016).

23. S. L. Jacques, "Optical properties of biological tissues: a review," Phys. Med. Biol. 58(11), R37-R61 (2013).

24. D. A. Baab, P. A. Oberg, and G. A. Holloway, "Gingival blood flow measured with a laser Doppler flowmeter," J. Periodontal. Res. 21(1), 73-85 (1986).

25. J. C. Ramirez-San-Juan et al., "Spatial versus temporal laser speckle contrast analysis in the presence of static optical scatterers," J. Biomed. Opt. 19(10), 106009 (2014).

26. P. Wilder-Smith et al., "In vivo imaging of oral mucositis in an animal model using optical coherence tomography and optical Doppler tomography," Clin. Cancer. Res. 13(8), 2449-2454 (2007).

Caitlin Regan received her BS degree in bioengineering with a focus in mechanics from the California Institute of Technology and her MS degree in biomedical engineering from the University of California, Irvine, where she is a PhD candidate in the Microvascular Therapeutics and Imaging Laboratory.

Sean M. White graduated from the University of Rochester with his $\mathrm{BS}$ degree in optics and received his $\mathrm{PhD}$ in biomedical engineering from the University of California, Irvine. His research is focused primarily on optically based hemodynamic measurement technology. In particular, he has broad experience developing laser speckle imaging instrumentation. He has cofounded several medical device companies that improve existing standards of care by leveraging novel noninvasive measurement techniques.

Bruce Y. Yang received his BS degree in bioengineering from the University of California, San Diego, California, USA, in 1996, and the $\mathrm{MS}$ and $\mathrm{PhD}$ degrees in biomedical engineering from the University of California, Irvine, California, USA, in 2009 and 2013, where he was a National Institutes of Health TL-1 predoctoral fellow. He currently is a specialist at the Beckman Laser Institute and a cofounder of a medical device company.

Thair Takesh graduated from Aleppo Dental School in Syria and joined the faculty at Aleppo University. After specialty training in periodontology at Cardiff University and completion of a master's degree in biomechanical engienering, he worked as a visiting scientist at Pennsylvania State University and now as a research scientist at the Beckman Laser Institute and Medical Clinic, University of California, Irvine.

Cherie Wink has been a practicing dental hygienist for more than 20 years. She is a clinician, dental hygiene educator, community outreach coordinator, and assistant research specialist at the University of California, Irvine Beckman Laser Institute. She has taught clinical sciences, special needs, oral pathology and National Board Review at Concorde College. His general research area is focused on methods for advancing dental practices and improving clinical outcomes.

Petra Wilder-Smith is a professor and director of dentistry at the University of California, Irvine, Beckman Laser Institute. Her research interests focus on novel optical approaches to oral diagnosis, especially precancerous and cancerous changes. Other research interests include oral biofilm, dental de- and remineralization, and pulpal vitality. Additional projects investigate use of stem cells to prevent or mitigate cancer-therapy-induced mucositis. He serves on many advisory and editorial boards, and is immediate past president of IADR Diagnostic Sciences Group.

Biographies for the other authors are not available. 